\title{
EDITORIAL
}

\section{Lie Algebras and Related Topics}

\author{
Yucai SU ${ }^{1}$, Shaobin TAN ${ }^{2}$, Hechun ZHANG ${ }^{3}$ \\ 1 Department of Mathematics, Tongji University, Shanghai 200092, China \\ 2 School of Mathematical Sciences, Xiamen University, Xiamen 361005, China \\ 3 Department of Mathematical Science, Tsinghua University, Beijing 100084, China
}

(C) Higher Education Press and Springer-Verlag Berlin Heidelberg 2011

From July 20 to 26, 2011, the Department of Mathematics at Huzhou Teachers College will host the 12th National Conference on Lie Algebras. Aiming to offer a forum for people working in this old but still exciting field to exchange research ideas and enhance collaborations, the conference will bring together some 150 participants from all over China and some participants overseas as well. There will be 41 talks during the conference and among them 8 are invited ones. Detailed information on the conference, including the organizing committee, the scientific committee, the invited speakers, and the conference program, can be found in

$$
\text { http://lxy.hutc.zj.cn/liealgebra/ }
$$

This special issue consists of one survey article and 13 research articles. All papers in this special issue have been refereed. We would like to thank all the referees for their helpful assistance. We wish to thank all the contributors for their generous support, and Frontiers of Mathematics in China, along with its managing editor Mr. Shannian Lu especially, who have helped us a lot on the publication.

We extend our thanks to the National Natural Science Foundation of China, the Science Foundation of Zhejiang Province, the Chinese Mathematical Society, the Department of Mathematics at Huzhou Teachers College and Professors Liu Dong and Liu Taishun for their generous financial supports.

\section{The 12th National Conference on Lie Algebras}

\section{Scientific Committee}

Zhang Hechun (Chairman)

Bai Chengming

Ding Xiangmao

\footnotetext{
Received May 31, 2011

E-mail: ycsu@ustc.edu.cn, tans@xmu.edu.cn, hzhang@math.tsinghua.edu.cn
} 
Jiang Cuibo

Peng Liangang

$\mathrm{Su}$ Yucai

Tan Shaobin

Xu Xiaoping

\section{Organizing Committee}

Liu Dong (Chairman)

Cao You'an

Deng Shaoqiang

Hu Naihong

Jin Quanqin

Lin Weiqiang

Liu Wende

Shu Bin

Song Guangai

Xin Bin

Zhu Linsheng 